\title{
Solid-State Structure of Comb-Like Polymers Having $n$-Octadecyl Side Chains I. Cocrystallization of Side Chain with $\boldsymbol{n}$-Octadecanoic Acid
}

\author{
Katsuhiro Inomata, Yoshiaki Sakamaki, Takuhei Nose, and Shintaro SaSaki* \\ Department of Polymer Chemistry, Tokyo Institute of Technology, 2-12-1 Ookayama, Meguro-ku, Tokyo 152, Japan \\ * Department of Chemical Materials Science, School of Materials Science, \\ Japan Advanced Institute of Science and Technology, \\ Asahidai, Tatsunokuchi, Ishikawa 923-12, Japan
}

(Received June 6, 1996)

\begin{abstract}
Solid-state structures of comb-like polyacrylates (PAx) and polymethacrylates (PMx) having crystallizable $n$-octadecyl side chains and their blends with $n$-octadecanoic acid (C18) have been investigated by means of differential scanning calorimetry and X-ray diffraction methods. It is concluded that $n$-alkyl side chains of comb-like polymer cocrystallize with low-molecular-weight $\mathrm{C} 18$ by incorporation of $\mathrm{C} 18$ into hexagonal crystalline lattice. In the case of amorphous PMx, the addition of C18 can induce a formation of crystalline domain by the cocrystallization with the side chain. Differences in the crystallization behavior, such as fractions of crystallized methylene units and effects of thermal history, between PAx and PM $x$ are found to be considerably large. These results are explained by the difference in the segmental mobility of main chain. KEY WORDS Comb-Like Polymer / $n$-Octadecanoic Acid / Cocrystallization / X-Ray Diffraction / DSC Measurement / $n$-Alkyl Side Chain /
\end{abstract}

Differences in solid-state nature between side chain and main chain in comb-like polymers are anticipated to give a wide variety of phases, structures, and morphologies, for example, those in side-chain liquid crystalline polymers ${ }^{1}$ and rigid-rod polymer with flexible side chains. ${ }^{2-4}$ Comb-like polymers, which have crystallizable long $n$-alkyl side chains being attached to amorphous main chain, are known to pack into layered structure with alternating crystalline side-chain region and amorphous main-chain region in the solid state. ${ }^{5-13}$ Platé and Shibaev ${ }^{6}$ studied these comb-like polymers extensively. X-Ray diffraction profile for such polymer has an intense reflection corresponding to the Bragg spacing $d$ of $4.2 \AA$, which suggests a formation of hexagonal crystalline lattice of $n$-alkyl side chain. There also exists a reflection in smaller angle region, which is considered to originate from the layered structure. The repeating distance of crystalline-amorphous layer can be obtained by the diffraction angle of this reflection. Platé et al. ${ }^{6}$ observed striking difference in the diffraction patterns and layer spacings between comb-like polyacrylates and polymethacrylates. In order to interpret this difference, they proposed two models for chain packing: two-layer structure and one-layer structure for respective polymers. In the former model, the side chains are extended on both side of the main chain and these polymers pack closely because of the flexibility of the main chain. In the latter structure, the disordered packing of the end groups of side chains occurs because of the increased bulkiness of the main chain, and the polymer chains are arranged with a shift toward the extended side-chain axis. Later, Hsieh et al. ${ }^{9}$ proposed a new structural model for the solid state poly $(n$-octadecyl methacrylate), where the side-chain crystallites are formed by intercalating side chains pointing in opposite directions. Although the detailed solid-state structures of these comb-like polymers have not been clarified as yet, the layered structure with the alternating segregation of side-chain and main-chain layer is commonly accepted. ${ }^{5-13}$ In this series of studies, we aim to investigate solid state nature of comb-like polymers comprising amorphous main chain and crystallizable side chains.

These polymers are used as pour-point depressants for lubricating oils or fuels. This depressant effect is considered to work by the ability of the polymers to cocrystallize with $n$-paraffins into the same crystalline lattice. ${ }^{14-19}$ Rubin and Pugliese ${ }^{15-18}$ investigated thermodynamical and structural changes of solidified $n$ paraffins containing poly( $n$-alkyl methacrylate)s. In the solid state of poly( $n$-octadecyl methacrylate) blended with $n$-dodecane and $n$-octacosane, side chains of the polymer were found to cocrystallize with $n$-hydrocarbons and to alter the solid-state structure of low-molecularweight component. From a viewpoint of thermally reversible gel formation, Platé and Shibaev ${ }^{6}$ studied the solution of comb-like polymers in solvent such as $n$ aliphatic hydrocarbons and alcohols. From these findings in literature, it is expected that the similarity of chemical and crystal structure between those of side chain of comb-like poiymer and $n$-aliphatic compound will lead their blends to various phases and morphologies in solid state.

In this paper, we focus our attention on cocrystallization behavior of blends of comb-like polymers with $n$-octadecanoic acid $\left(\mathrm{CH}_{3}\left(\mathrm{CH}_{2}\right)_{16} \mathrm{COOH}, \mathrm{C1}\right)$. As the comb-like polymers, we adopt polymethacrylates and polyacrylates having $n$-octadecyl residue as the side chain: statistically random copolymer poly [(methyl methacrylate)-stat-( $n$-octadecyl methacrylate) $]$ and poly[(methyl acrylate)-stat-( $n$-octadecyl acrylate)]. The $n$-alkyl fatty acid $\mathrm{C} 18$ has chemical structure similar to that of the side chain of polymers, and crystallizes at a temperature higher than crystallization temperatures of polymers. Influences of main-chain structure, composition of the $n$-octadecyl residue in pure polymers, and blend composition upon the phase behavior are systematically studied. Morphological changes accompanied with 
these factors will be reported in detail in Part II of this series. $^{20}$

\section{EXPERIMENTAL}

\section{Materials}

Methyl acrylate and methyl methacrylate were treated with aqueous alkali to remove inhibitor, dried, and distilled before use. $n$-Octadecyl acrylate, $n$-octadecyl methacrylate, and $n$-octadecanoic acid (C18) were commercially available and used without purification. Polymerizations and random copolymerizations ${ }^{7,9}$ of the above monomers were carried out in a solution of toluene at $60^{\circ} \mathrm{C}$ for three days with the use of $0.5 \mathrm{~mol} \%$ azobisisobutyronitrile as initiator. The reacted mixtures were poured into acetone or hot methanol. The precipitated polymers were washed with acetone or hot methanol till free of monomers. The $n$-octadecyl-sidechain compositions of the copolymers were determined by ${ }^{1} \mathrm{H}$ NMR spectra. Hereafter, a polymer which contains $x \mathrm{~mol} \%$-octadecyl ester residue is designated as PA $x$ for polyacrylates and PM $x$ for polymethacrylates. Sample codes and molecular weights of the prepared samples are listed in Table I. In cooling scan of differential scanning calorimetry (DSC) measurement for these polymers from the molten state, a sharp exothermic peak was observed for all the series of polyacrylates and for polymethacrylates of high $n$-octadecyl side-chain composition: PA100, PA70, PA54, PA33, PM100, PM71, and PM53. These polymers also exhibited a sharp X-ray diffraction peak at wide-angle region, which suggests the formation of hexagonally-packed crystallite of the $n$-alkyl side chains. On the other hand, PM43 and PM16 showed no transition peak in DSC thermogram and no crystalline diffraction in X-ray profile.

The blend samples of comb-like polymer with C18 were prepared as follows. The weighted polymer and fatty acid were dissolved in tetrahydrofuran (THF), casted on Teflon sheet, heated at $90^{\circ} \mathrm{C}$, quenched to room temperature, and dried in vacuum. Blends of high fatty acid content were ground to fine powder in order to prepare homogeneously-mixed samples. The compositions of the blends are expressed by the mole fraction of the monomeric unit of polymer and C18 molecule.

\section{Methods}

DSC thermograms were recorded by using a Perkin-

Table I. Characteristics of sample polymers

\begin{tabular}{llcc}
\hline & Code & $\begin{array}{c}\text { Fraction of octadecyl } \\
\text { ester residue } / \mathrm{mol}^{\mathrm{a}}{ }^{\mathrm{a}}\end{array}$ & $M_{w}{ }^{\mathrm{b}}$ \\
\hline Polyacrylates & PA100 & 100 & 190000 \\
& PA70 & 70 & 160000 \\
& PA54 & 54 & 150000 \\
& PA33 & 33 & 140000 \\
Polymethacrylates & PM100 & 100 & 440000 \\
& PM71 & 71 & 230000 \\
& PM53 & 53 & 170000 \\
& PM43 & 43 & 120000 \\
& PM16 & 16 & 70000 \\
\hline
\end{tabular}

${ }^{a}$ Determined by ${ }^{1} \mathrm{H}$ NMR spectra.

${ }^{b}$ Determined by SEC analyses (calibrated by polystyrene).
Elmer DSC model II instrument, and indium was used as the reference for correcting heat of transition. XRay diffraction measurements were performed with Nifiltered $\mathrm{Cu}-K \alpha$ radiation (wave length $\lambda=1.5418 \AA$ ) generated from RU-200BH (Rigaku Co.) and an imaging plate detector (R-AXIS IID, Rigaku Co.). All measurements were carried out at room temperature. The distance between the sample and the imaging plate was calibrated by the 111 reflection of silicon powder sprinkled over the sample. Diffraction diagram recorded on the flat imaging plate by normal-beam-transmission technique was converted to the one-dimensional profile against diffraction angle $2 \theta$. The Bragg spacing $d$ was given by the equation $d=\lambda /(2 \sin \theta)$. Size-exclusion chromatography (SEC) was run on Toyo-Soda HLC 803 using THF as eluent. The columns were calibrated with linear narrow molecular weight polystyrenes.

\section{RESULTS AND DISCUSSION}

\section{Cocrystallization of C18 with $n$-Octadecyl Side Chain of $P A x$ and $P M x$}

Figure 1 shows the composition-dependence of DSC thermograms of the blend system of PA54/C18 measured by cooling with a rate of $20 \mathrm{deg} \mathrm{min}^{-1}$ from melt state. A peak corresponding to side-chain crystallization is observed at $35^{\circ} \mathrm{C}$ for the pure PA54. There is also one exothermic peak for the blends with less than $16 \mathrm{~mol} \%$ C18 content. For the samples of higher $\mathrm{C} 18$ contents, another exothermic peak is observed at higher-temperature region. With the increase of $\mathrm{C} 18$ content, the position of this peak moves toward that of pure $\mathrm{C} 18$ $\left(62^{\circ} \mathrm{C}\right)$. Similar thermograms are also obtained for the blends of the other side-chain crystallizable polymers (see Experimental) with C18. Exceptionally, the thermograms of PA100/C18 system show some complicated behavior. Details will be described in Part II of this series. ${ }^{20}$

X-Ray diffraction measurements of the PA54/C18 blend samples cooled rapidly from the melt were carried out at room temperature. Thus prepared samples were confirmed to exhibit nearly identical DSC heating thermogram with that for the sample cooled at the rate of $20 \mathrm{deg} \mathrm{min}^{-1}$ in DSC instrument. Results are shown in Figure 2. In the diagram of pure PA54 (bottom of Figure 2), two intense reflections are observed at diffraction angles $2 \theta=2.7^{\circ}$ and $21.5^{\circ}$. The former corre-

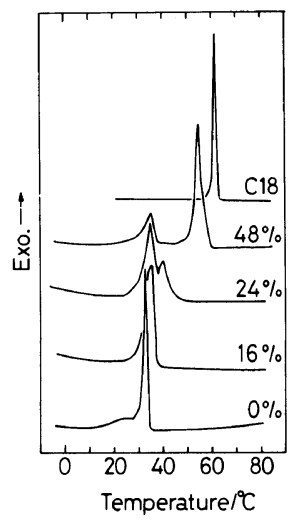

Figure 1. DSC cooling thermograms for PA54/C18 systems with $\mathrm{C} 18$ contents of $0,16,24,43$, and $100 \mathrm{~mol} \%$, measured at the rate of $20 \mathrm{deg} \min ^{-1}$. 


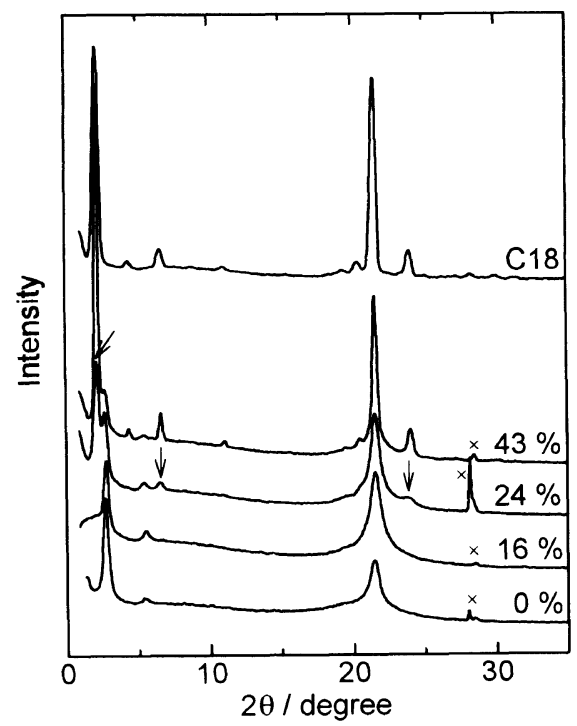

Figure 2. X-Ray diffraction diagrams for $\mathrm{PA} 54 / \mathrm{C} 18$ systems with C18 contents of $0,16,24,43$, and $100 \mathrm{~mol} \%$, measured at room temperature. The baseline for each diagram is shifted up and down in order to avoid overlapping. The peaks designated by $\times$ correspond to those of silicon sprinkled on the sample.

sponds to the layered structure of the alternating crystalline side-chain region and amorphous region, and the latter to hexagonal packing of $n$-alkyl chain. In the diffraction patterns of blends with $16 \mathrm{~mol} \% \mathrm{C} 18$ content or less, only these two peaks are observed with varying their relative intensities: addition of $\mathrm{C} 18$ causes decrease in intensity of the reflection at smaller angle and increase at wider angle. For samples containing more than $24 \mathrm{~mol} \% \mathrm{Cl}$, some additional peaks appear and increase in intensity with $\mathrm{C} 18$ content (indicated by the arrow in Figure 2). At the top of Figure 2, the diffraction pattern of pure $\mathrm{C} 18$ is presented. It is known that $\mathrm{C} 18$ molecule crystallizes into some polymorphs. ${ }^{21-24}$ From the intensity and spacing of each observed reflection, the crystalline form of $\mathrm{C} 18$ prepared in this work is considered to be of C-form. ${ }^{22}$ Comparison of profiles of the pure $\mathrm{C} 18$ and blend PA54/C18 containing more than $24 \mathrm{~mol} \% \mathrm{Cl} 8$ indicates that the newly appeared peaks can be assigned to the crystallite in $\mathrm{C}$-form of $\mathrm{C} 18$. From these observations, we may say that there exists only hexagonally-packed crystalline lattice in the sample containing less than $16 \mathrm{~mol} \% \mathrm{C} 18$, which is suggested by the absence of the X-ray diffraction peaks and DSC transition peaks corresponding to pure C18. In the blends containing more than $24 \mathrm{~mol} \% \mathrm{Cl} 18$, the isolated $\mathrm{C} 18$ crystallites are coexisting with the hexagonal crystalline lattice. Similar X-ray diffraction profiles are also observed in the blends comprising another side-chain crystallizable polymer and small amount of $\mathrm{C} 18$.

Figure 3 shows DSC thermograms of PM43 and its blends with $\mathrm{C} 18$ measured at the cooling rate of 20 $\operatorname{deg} \min ^{-1}$. Absence of any crystallization peaks in the thermogram of pure PM43 indicates that this comblike polymer do not form crystalline domain in the solid state. However, its blend of $7 \mathrm{~mol} \% \mathrm{C} 18$ content gives a transition peak at $\sim 10^{\circ} \mathrm{C}$, which is nearly the crystallization temperature of pure PM53. In the X-ray

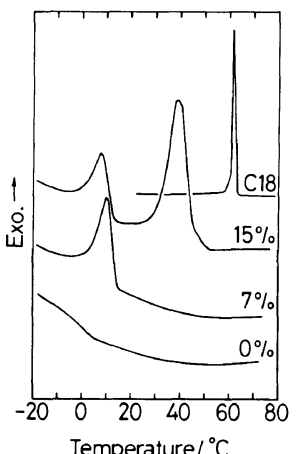

Figure 3. DSC cooling thermograms for $\mathrm{PM} 43 / \mathrm{C} 18$ systems with $\mathrm{C} 18$ contents of $0,7,15$, and $100 \mathrm{~mol} \%$, measured at the rate of $20 \mathrm{deg} \min ^{-1}$.

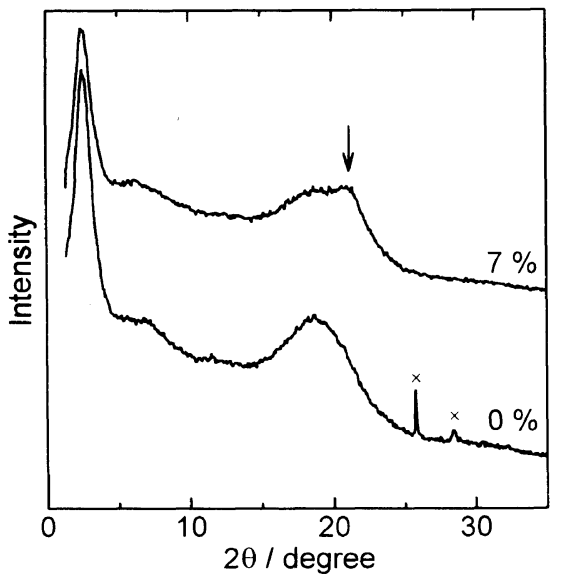

Figure 4. X-Ray diffraction diagrams of $\mathrm{PM} 43$ and $\mathrm{PM} 43 / \mathrm{C} 18$ blend with $7 \mathrm{~mol} \% \mathrm{C} 18$ content, measured at room temperature. The baseline for each diagram is shifted up and down in order to avoid overlapping. The peaks designated by $x$ correspond to those of silicon sprinkled on the sample.

diffraction diagram of pure PM43 (Figure 4), there can be seen amorphous halo around $2 \theta=18^{\circ}$, and intense but broad peak at smaller angle region which is considered to originate from the correlation between the segregated side chain and main chain. For PM43/C18 blend with $7 \mathrm{~mol} \% \mathrm{C} 18$, there exists a weak shoulder peak at $2 \theta=21^{\circ}$ (indicated by the arrow in Figure 4) which can be assigned to the hexagonal packing of $n$-alkyl chain. These observations suggest that the addition of C18 induces the crystallization of the side chain for the amorphous comb-like polymers. Similar results are also observed in PA16/C18 system.

For the blends which give a single crystallization peak in the DSC cooling thermogram, we calculate heat of crystallization for one mole of octadecyl side chain, $\Delta H_{\text {side }} . \Delta H_{\text {side }}$ will not change with the C18 content if the added $\mathrm{C} 18$ does not influence the crystallization behavior of the side chain. These values are plotted against the mole fraction of $\mathrm{C} 18$ in Figure 5. The increase of $\Delta H_{\text {side }}$ with $\mathrm{C} 18$ content indicates the increase of the number of methylene unit in the hexagonally-packed crystalline domain. It can be pointed out that the blended C18 molecules are incorporated into the crystalline lattice formed by the side chains of polymer when amount of the added C18 is small, that is to say, C18 molecules are cocrystallizing with $n$-octadecyl side chains in solid state. 


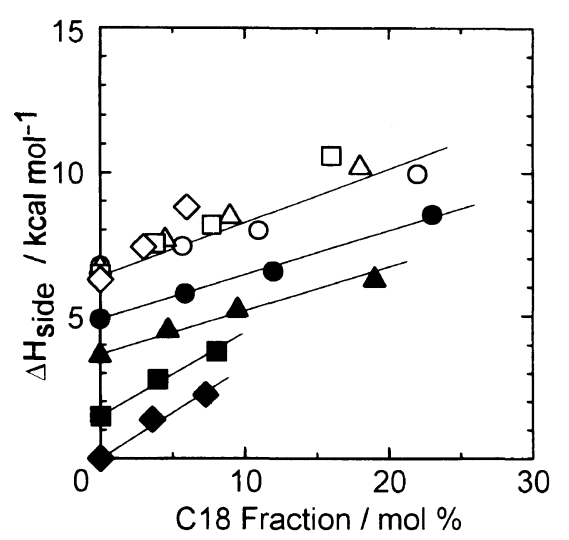

Figure 5. Plots of the heat of crystallization per mole of $n$-octadecyl side chain, $\Delta H_{\text {side }}$, against the mole fraction of $\mathrm{C} 18$ in the blends

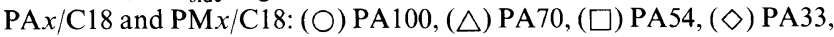

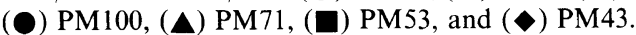

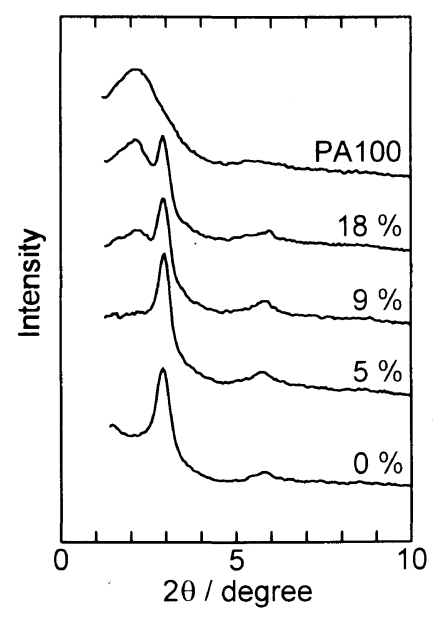

Figure 6. X-Ray diffraction diagrams for PA100 and PA70/C18 systems with $\mathrm{C} 18$ contents of $0,5,9$, and $18 \mathrm{~mol} \%$, measured at room temperature. The baseline for each diagram is shifted up and down in order to avoid overlapping.

Figure 6 is the X-ray diffraction diagrams in smallangle region for pure PA100 and blends of PA70/C18. There is a sharp reflection at $2 \theta=2.9^{\circ}(d=30 \AA)$ in the diagram of pure PA70, while PA100 gives a somewhat broad peak at $2 \theta=2.1^{\circ}(d=42 \AA)$. As seen in the figure, the scattering intensity around $2.1^{\circ}$ observed in the profile of PA70/C18 blend is gradually increased with the $\mathrm{C} 18$ content. For the samples containing $\mathrm{C} 18$ to the extent of $18 \mathrm{~mol} \%$, no evidence of crystallization of isolated C18 is recognized in DSC thermogram and wide-angle $\mathrm{X}$-ray profile. Thus, the reflection at $2.1^{\circ}$ for the blend sample do not suggest the formation of $\mathrm{C} 18$ crystallite but the formation of the layered structure observed in PA100. This result means that the cocrystallization of C18 with the side-chain crystalline lattice and the increase of the composition of side chain of comb-like polymer have a similar effect on the packing manner of the chain. Figure 6 also suggests the coexistence of two kinds of layered structures in PA70/C18 blends, one is observed in PA70, and the other in PA100. This observation indicates that the arrangement of the crystalline and amorphous layers varies with the fraction of $n$-alkyl chain in the system. More detailed discussion concerning with the layered structure will be presented

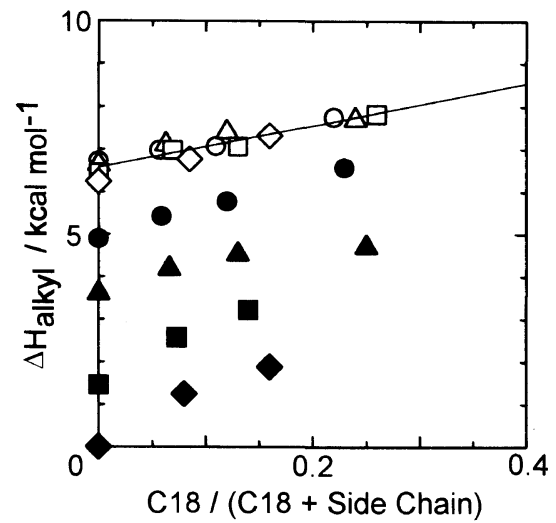

Figure 7. Plots of $\Delta H_{\text {alkyl }}$, the heat of crystallization per mole of $n$-alkyl chain including $n$-octadecyl side chain and C18, against the fraction of $\mathrm{C} 18$ in all $n$-alkyl chain $(=\mathrm{C} 18 /(\mathrm{C} 18+$ side chain $))$. Symbols are the same as in Figure 5.

\section{in Part II of this series. ${ }^{20}$}

\section{Effect of Main-Chain Structure}

In the preceding section, we obtained the heat of crystallization per mole of $n$-octadecyl side chain, $\Delta H_{\text {side }}$, for the blend samples. From the increase of $\Delta H_{\text {side }}$ with $\mathrm{C} 18$ content, we have suggested the cocrystallization of the comb-like polymer with C18. In order to consider the state of the crystalline domain containing both $n$-octadecyl side chain and $\mathrm{C} 18$, we calculate $\Delta H_{\text {alkyl }}$, which is defined as the heat of crystallization for one mole of $n$-alkyl chain: including both $n$-octadecyl side chain and C18 molecule. The values of $\Delta H_{\text {alkyl }}$ are plotted against the fraction of $\mathrm{C} 18$ in the total $n$-alkyl chain $(\mathrm{C} 18 /(\mathrm{C} 18+$ side chain $))$ in Figure 7. As shown in this figure, $\Delta H_{\text {alkyl }}$ is increased with the increase of $\mathrm{C} 18$ content for both PM $x$ and PAx series. This result suggests that the cocrystallization behavior of comb-like polymer with $\mathrm{C} 18$ increases the averaged number of methylene unit, which existing in the hexagonally-packed crystalline domain, in one $n$-alkyl chain. In the series of PAx (open symbols in Figure 7), all data points can be expressed by a single line. Extrapolation of this line to $100 \%$ of $\mathrm{C} 18$ composition gives the value of $\Delta H_{\mathrm{alkyl}}$ as $11.5 \mathrm{kcal} \mathrm{mol}^{-1}$. Heat of melting for the hexagonal modification of the $n$-alkane has been reported as 0.73 kcal per mole of methylene unit, ${ }^{8,25}$ then heat of transition for sixteen methylenes can be calculated as 11.7 $\mathrm{kcal} \mathrm{mol}{ }^{-1}$, which is comparable with the extrapolated value of $\Delta H_{\text {alkyl }}$.

In the case of the series of $\mathrm{PM} x, \Delta H_{\text {alkyl }}$ varies with the change of $x$ as shown by the filled symbols in Figure 7, which is different from the case of PAx. This tendency has been explained by difference in the mobility of the segmental motion of the main chain. ${ }^{8}$ Melting temperature of the hexagonally-packed $n$-alkyl side chain is $10-40^{\circ} \mathrm{C}$, which is higher and lower than the glass transition temperature, $T_{\mathrm{g}}$, of poly(methyl acrylate) and poly(methyl methacrylate), respectively. Thus, the segmental motion of the main chain of PM $x$ may be considerably restricted at the crystallization temperature of $n$-alkyl side chain. In the case of PAx series, the flexibility of the main chain may facilitate the crystallization of the side chains.

In order to examine the effect of the main chain upon 


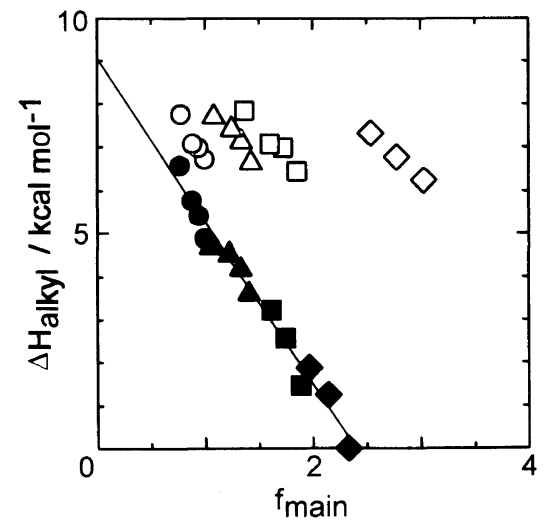

Figure 8. Plots of $\Delta H_{\text {alkyl }}$ against $f_{\text {main }}$, which is defined as the ratio of the number of repeating unit of the main chain to the number of the $n$-alkyl chain. Symbols are the same as in Figure 5.

the crystallization behavior of the side chain, we define the ratio of the number of repeating unit of the main chain $\left(-\mathrm{CH}_{2}-\mathrm{CH}-\right.$ for $\mathrm{PAx}$ and $-\mathrm{CH}_{2}-\mathrm{C}\left(\mathrm{CH}_{3}\right)-$ for PM $x$ ) to the number of $n$-alkyl chain (both $n$-octadecyl side chain and C18 molecule) as $f_{\text {main }}$. The value of $f_{\text {main }}$ represents a relative amount of main-chain group against the crystallizable $n$-alkyl chain. As shown in Figure 8, the series of PM $x$ have a linear relation between $f_{\text {main }}$ and $\Delta H_{\text {alkyl }}$, which is a contrast to PAx series represented by the open symbols. This figure suggests that, for $\mathrm{PM} x$, the crystallization of $n$-alkyl chain are under strong influence of the existence of the main chain. The extrapolated value of $\Delta H_{\text {alkyl }}$ to $f_{\text {main }}=0$ is $9.1 \mathrm{kcal}$ $\mathrm{mol}^{-1}$, which is a little smaller than the calculated heat of fusion of sixteen hexagonally-packed $\mathrm{CH}_{2}$ groups $\left(11.7 \mathrm{kcal} \mathrm{mol}^{-1}\right)$. From Figures 7 and 8 , we can say that the crystallization behavior of $n$-alkyl chain is nearly independent of the presence of the main chain for PAx, and for PMx, the main chain strongly influences the crystallization behavior of $n$-alkyl chain.

\section{Effect of Thermal History}

In order to investigate the manner of cocrystallization of $\mathrm{C} 18$ with the side chain, we have measured DSC heating thermogram of blends which have been undergone different thermal history. In the cooling thermogram of blend of PM $100 / \mathrm{C} 18$ with $23 \mathrm{~mol} \% \mathrm{C} 18$ content measured at the rate of $20 \mathrm{deg} \min ^{-1}$ from the melt state, there appears a single exothermic peak which corresponds to the cocrystallization of the side chain with C18. The curve a in Figure 9 is the heating thermogram of this quenched sample, measured at the rate of $10 \mathrm{deg} \min ^{-1}$. Just behind a sharp endothermic peak at $41^{\circ} \mathrm{C}$, an exothermic peak exists, and after that, second endothermic peak is observed at $63^{\circ} \mathrm{C}$. The endothermic peaks at lower and higher temperature are considered as the melting of the hexagonally-cocrystallized domain and the isolated $\mathrm{C} 18$ crystallite, respectively. Curve $\mathrm{b}$ in Figure 9 is the heating thermogram of the blend with the same composition, which has been first quenched from the molten state, annealed at $54^{\circ} \mathrm{C}$ (the middle point of the two endothermic peaks) for a few minutes, and subsequently cooled to $-13^{\circ} \mathrm{C}$ with the rate of $5 \mathrm{deg} \min ^{-1}$. In $\mathrm{b}$, the exothermic peak between the two endothermic peaks can not be observed, and peak

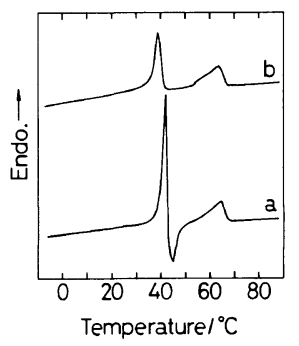

Figure 9. DSC heating thermograms for PM100/C18 system with $23 \mathrm{~mol} \% \mathrm{C} 18$ measured at the rate of $10 \mathrm{deg} \mathrm{min}^{-1}$. Sample for (a) is rapidly cooled $\left(20 \mathrm{deg} \mathrm{min}^{-1}\right)$ from molten state, and sample for (b) is obtained by annealing the rapidly cooled sample at $54{ }^{\circ} \mathrm{C}$.

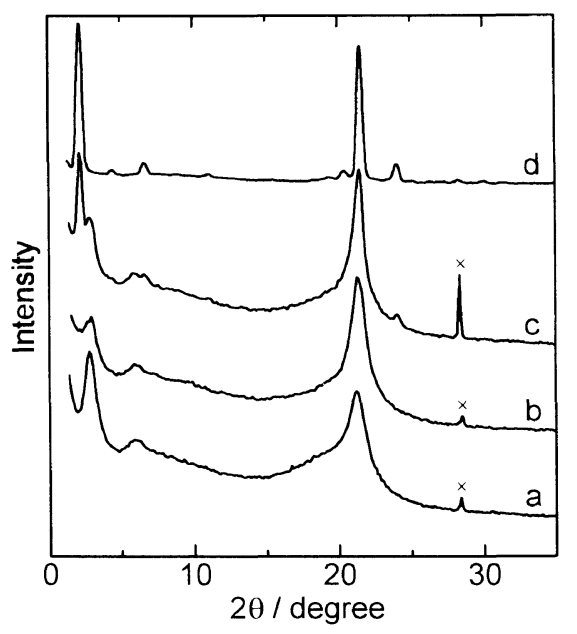

Figure 10. X-Ray diffraction diagrams measured at room temperature. (a) PM 100, (b) PM 100/C18 with 23 mol\% C18 content crystallized from molten state by the cooling rate of $20 \mathrm{deg} \mathrm{min}^{-1}$, (c) the same composition as (b) but crystallized after annealing at $54^{\circ} \mathrm{C}$, and (d) $\mathrm{C} 18$. The baseline for each diagram is shifted up and down in order to avoid overlapping. The peaks designated by $\times$ correspond to those of silicon sprinkled on the sample.

area of the endothermic peak at lower temperature is decreased.

The X-ray diffractograms for the samples undergone the identical thermal treatments as mentioned above are shown in Figure 10. All the measurements have been conducted at room temperature. In the diagram of the quenched sample (Figure 10b), there are only two diffraction peaks as that of pure PM100 (Figure 10a), which suggests the occurrence of the cocrystallization. Figure 10c shows the profile of the sample annealed at $54^{\circ} \mathrm{C}$. There exist some additional reflections such as at $2 \theta=2^{\circ}$ and $24^{\circ}$, and these can be assigned to the pure C18 crystallite shown in Figure 10d. These variations in the thermogram and X-ray profile depending on the thermal history are also observed for the blends of other side-chain crystallizable $\mathrm{PM} x$, but negligible for the blends of PAx series.

These phenomena give information concerning the manner of the cocrystallization of $\mathrm{C} 18$ with the side chain. When the blend of polymethacrylate with $\mathrm{C} 18$ is cooled rapidly, the formation of pure C18 crystallite may be considerably prohibited because of the rapid cooling and the restricted segmental motion of the main chain, and the cocrystallization of side chain may occur with excess amount of $\mathrm{C} 18$ molecules as compared with the equilibrium condition. Heating of this solid cause 
the melting of the cocrystallized domain, and the incorporated excess $\mathrm{C} 18$ molecules become free and crystallize by themselves again because the temperature is lower than the melting point of pure C18. The crystallization of $\mathrm{C} 18$ gives the exothermic peak in the heating thermogram as seen in Figure 9a. In the subsequent cooling scan from $54^{\circ} \mathrm{C}$, the side chains cocrystallize with less amount of $\mathrm{C} 18$ because some $\mathrm{C} 18$ crystallites have already been formed during the annealing. As a result, the area under the melting peak at lower temperature is decreased and the exothermic peak disappears in the subsequent heating thermogram (Figure 9b). In the case of the series of polyacrylates, the segmental mobility of the main chain is higher than that of polymethacrylates, and the crystallization of $\mathrm{C} 18$ may not be severely hindered even in the rapid-cooling process. The difference between the thermogram of rapidly-cooled PM $x$ blend and that of the annealed one suggests the occurrence of the cocrystallization of $\mathrm{C} 18$ with the side chains.

\section{CONCLUDING REMARKS}

Solid-state structures of the blends of comb-like polymers having $n$-octadecyl side chains (PA $x$ and PM $x$ ) with $n$-octadecanoic acid $(\mathrm{C} 18)$ are investigated by means of DSC and X-ray diffraction methods. PM $x$ having more than $43 \mathrm{~mol} \% n$-octadecyl ester residue and all PA $x$ are crystalline polymers, in which side chains form hexagonally-packed crystalline lattice. The side chains of these polymers are cocrystallizing with $\mathrm{C} 18$ by incorporation of $\mathrm{C} 18$ into the crystalline lattice of the side chain. Although the pure PM43 and PM16 can not form crystalline domain, the addition of $\mathrm{C} 18$ to these polymers induces the formation of hexagonal crystallite by the cocrystallization of $\mathrm{C} 18$ with the side chain. The cocrystallization phenomenon increases the number of methylene unit which existing in the crystalline domain, and changes the crystalline-amorphous layered structure as seen in PA70/C18 system. The difference in the crystallization behavior, i.e., the $\mathrm{C} 18$-content dependence on $\Delta H_{\text {alkyl }}$ or the effect of thermal history, between PAx and PM $x$ is large, probably because of the difference in the segmental mobility of the main chain. These systematic studies indicate a possibility to control the solid-state nature of the side-chain crys- tallizable comb-like polymers and their blends with $n$-alkyl chain. In Part II of this series, ${ }^{20}$ we will report a possibility of the control of the layered structure in solid state for these systems.

\section{REFERENCES}

1. V. P. Shibaev and N. A. Platé, Adv. Polym. Sci., 60/61, 173 (1984); V. P. Shibaev, Ya. S. Freidzon, and S. G. Kostromin, in "Liquid Crystalline and Mesomorphic Polymers," V. P. Shibaev and L. Lam, Ed., Springer-Verlag, New York N.Y., 1994, p. 77.

2. M. Ballauff, Angew. Chem. Int. Ed. Eng., 28, 253 (1989).

3. J. Watanabe, H. Ono, I. Uematsu, and A. Abe, Macromolecules, 18, 2141 (1985).

4. J. Watanabe, B. R. Harkness, M. Sone, and H. Ichimura, Macromolecules, 27, 507 (1994).

5. S. A. Greenberg and T. Alfrey, J. Am. Chem. Soc., 76, 6280 (1954).

6. N. A. Platé and V. P. Shibaev, J. Polym. Sci., Macromol. Rev., 8, 117 (1974).

7. E. F. Jordan, Jr., D. W. Feldeisen, and A. N. Wrigley, J. Polym. Sci., A-1, 9, 1835 (1971).

8. E. F. Jordan, Jr., B. Artymyshyn, A. Speca, and A.N. Wrigley, J. Polym. Sci., A-1, 9, 3349 (1971).

9. H. W. S. Hsieh, B. Post, and H. Morawetz, J. Polym. Sci., Polym. Phys. Ed., 14, 1241 (1976).

10. J. M. Barrales-Rienda, F. Fernandez-Martin, C. R. Galicia, and M. S. Chaves, Makromol. Chem., 184, 2643 (1983).

11. K. Yokota, T. Kougo, and T. Hirabayashi, Polym. J., 15, 891 (1983); K. Yokota and T. Hirabayashi, Polym. J., 18, 177 (1986); T. Hirabayashi and K. Yokota, Polym. J., 19, 1115 (1987).

12. L. J. Mathias, Polym. Commun., 29, 352 (1988).

13. P. L. Magagnini, E. L. Tassi, F. Andruzzi, and M. Paci, Polym. Sci., 36, 1502 (1994).

14. E. F. Jordan, Jr., S. Smith, Jr., R. D. Zabarsky, and A. N. Wrigley, J. Appl. Polym. Sci., 22, 1509 (1978); 1529 (1978); 1547 (1978).

15. I. D. Rubin, J. Appl. Polym. Sci., 36, 445 (1988).

16. I. D. Rubin and R. D. Pugliese, Angew. Makromol. Chem., 171, 165 (1989).

17. R. D. Pugliese and I. D. Rubin, Macromol. Rep., A28(Suppl. 2), 111 (1991).

18. R. D. Pugliese and I. D. Rubin, Polym., Prepr., Am. Chem. Soc., Div. Polym. Chem., 33, 340 (1992).

19. A. K. Chatterjee, S. D. Phatak, P. S. Murthy, and G. C. Joshi, J. Appl. Polym. Sci., 52, 887 (1994).

20. K. Inomata, S. Sakamaki, T. Nose, and S. Sasaki, Polym. J., 28 992 (1996).

21. K. Larsson and E. Sydow, Acta Chem. Scand., 20, 1203 (1966).

22. V. Malta, G. Celotti, R. Zannetti, and A. F. Martelli, J. Chem. Soc. (B), 548 (1971).

23. M. Goto and E. Asada, Bull. Chem. Soc. Jpn., 51, 2456 (1978)

24. M. Gotoh, J. Jpn. Oil Chem. Soc., 36, 909 (1987).

25. M. G. Broadhurst, J. Res. Nat. Bur. Stand., 66A, 241 (1962). 